\title{
Pathophysiological correlates of schizophrenia and incidental cerebral periventricular leukomalacia through a patient
}

\author{
Seda Sarikaya ${ }^{1 \oplus}$, Hasan Gokcay $^{2 \oplus}$, Yasin Hasan Balcioglu ${ }^{3 \oplus}$ \\ 'Istanbul Medeniyet University Faculty of Medicine, Department of Psychiatry, Istanbul - Turkey \\ 2University of Health Sciences, Bagcilar Training and Research Hospital, Department of Psychiatry, Istanbul - Turkey \\ ${ }^{3}$ Bakirkoy Prof. Mazhar Osman Training and Research Hospital for Psychiatry, Neurology, and Neurosurgery, Forensic Psychiatry \\ Unit, Istanbul - Turkey
}

Dear Editor,

Schizophrenia is a complex behavioral and cognitive syndrome thought to be caused by impaired brain development caused by genetic or environmental factors or both. (1). While examining both structural and functional brain abnormalities to demonstrate the etiology of this multifactorial disorder, some consistent findings have been reported to some extent, such as diffuse gray and white matter deficits and enlarged lateral ventricles (2-4). Periventricular leukomalacia (PVL) is a neurostructural change caused by periventricular white matter ischemia and since it mostly occurs in infants exposed to perinatal complications, studies are linking this early-acquired lesion with some neuropsychiatric diseases, including psychotic disorders (5). Although PVL is suggested to be one of the structural brain abnormalities potentially associated with schizophrenia through ventricular expansion, it remains unclear how PVL causes brain circuit disruptions that lead to schizophrenia (6). Below, we reported a 23-year-old male patient diagnosed with schizophrenia with an incidental lesion compatible with PVL on cranial magnetic resonance imaging, and we aimed to highlight the potential role of
PVL in etiopathogenesis schizophrenia. A 23-year-old male patient diagnosed with schizophrenia two years ago, was admitted to the psychiatry outpatient clinic auditory hallucinations, persecutory delusions, and associated social withdrawal symptoms. He demonstrated agitated behavior related to auditory hallucinations that ordered him to harm and kill himself, hence, he was taken to the inpatient unit. His initial complaints were social withdrawal and elementary auditory hallucinations which emerged three years ago. Following an insidious prodromal phase, he had presented with disorganized and inappropriate behaviors including walking outside naked, and command auditory hallucinations within a year, and $4 \mathrm{mg} / \mathrm{d}$ risperidone p.o. was started on his first and only psychiatric admission with a diagnosis of schizophrenia 18 months ago. At the present admission, the Positive and Negative Syndrome Scale (PANSS) total score was 112 . His caregivers claimed that he was noncompliant with antipsychotic treatment for at least a year, hence, they were unable to observe any improvement with regular treatment. The patient had no previous history of neurological diagnosis, no previousalcohol or substance abuse, and substance use was excluded through urine sampling. His mental state

How to cite this article: Sarikaya S, Gokcay H, Balcioglu YH. Pathophysiological correlates of schizophrenia and incidental cerebral periventricular leukomalacia through a patient. Dusunen Adam The Journal of Psychiatry and Neurological Sciences 2021;34:107-109.

Correspondence: Yasin Hasan Balcioglu, Bakirkoy Prof. Mazhar Osman Training and Research Hospital for Psychiatry, Neurology, and Neurosurgery, Forensic Psychiatry Unit, Istanbul - Turkey

E-mail: yhasanbalcioglu@gmail.com

Received: January 21, 2021; Revised: January 31, 2021; Accepted: February 04, 2021 
examination findings were as follows: he was cooperative and oriented, lacked verbal communication, and avoided eye contact, perseveration, commanding auditory hallucinations and persecutory delusions were noted. He had no insight. There was no pathological abnormality in physical and neurological examinations. Detailed blood screening, including hemogram and liver/renal functions, was within the normal range. A cranial magnetic resonance imaging (MRI) was performed as a routine admission protocol for inpatients. Multiple hyperintense lesions consistent with PVL located in the periventricular area of both lateral ventricles were reported, while mild bilateral cortical atrophy was observed (Fig. 1). According to the patient's early medical history, there were no perinatal complications described by his parents. The patient was started on risperidone $2 \mathrm{mg} / \mathrm{d}$ p.o. and gradually increased to 8 $\mathrm{mg} / \mathrm{d}$ over three weeks. Auditory hallucinations started to improve on the tenth day of the treatment, while persecutory delusions started to resolve in the second week. Psychomotor agitation improved. The PANSS total score dropped to 74 on the $20^{\text {th }}$ day of the treatment. He was discharged with $\mathrm{r} 8 \mathrm{mg}$ oral risporidone daily and monthly admission to the outpatient clinic was recommended. Although the exact etiology of schizophrenia is still unclear, it has been suggested that the disorder is associated with a multifactorial etiology including abnormal brain structures, genetics, viral infections, and immune diseases (1). Early structural brain changes have been linked to the occurance of schizophrenia due to permanently impaired neural circuits (2). PVL, which is primarily caused by perinatal damage of the developing brain, is associated with perinatal adversities including very preterm birth, and the risk of psychiatric disorders increases in individuals with perinatal complications (5). Ventricular enlargement led by PVL is associated with white matter damage, abnormal neurodevelopment, and disrupted neural networks that maintain appropriate cognitive, emotional, and behavioral functions $(7,8)$. Furthermore, it has been suggested that the increased release of proinflammatory cytokines related to perinatal immune activation caused by infections, hypoxia, or premature birth may lead to PVL, white matter damage, and ventricular dilation, all of which are included in the etiopathogenesis of schizophrenia (9-12). In particular, perinatal hypoxia has been suggested to cause selective long-term disturbances of the dopaminergic systems in experimental models (13). On the other hand, PVL interferes with the regular stratification of the cortex and causes a failure in normal brain development early in life. PVL and other white matter lesions are mainly repaired by Cajal-Retzius cells, however, the survival of these morphologically and molecularly distinct types of neurons on the surface of the developing cerebral cortex has been found to reduce in schizophrenia patients, particularly in young adulthood (11), indicating the presence of an insufficient neuronal regeneration process in schizophrenia. Strikingly, perinatal hypoxia and neuroinflammation, which are well-known causes of PVL, are also associated with schizophrenia independent of white matter
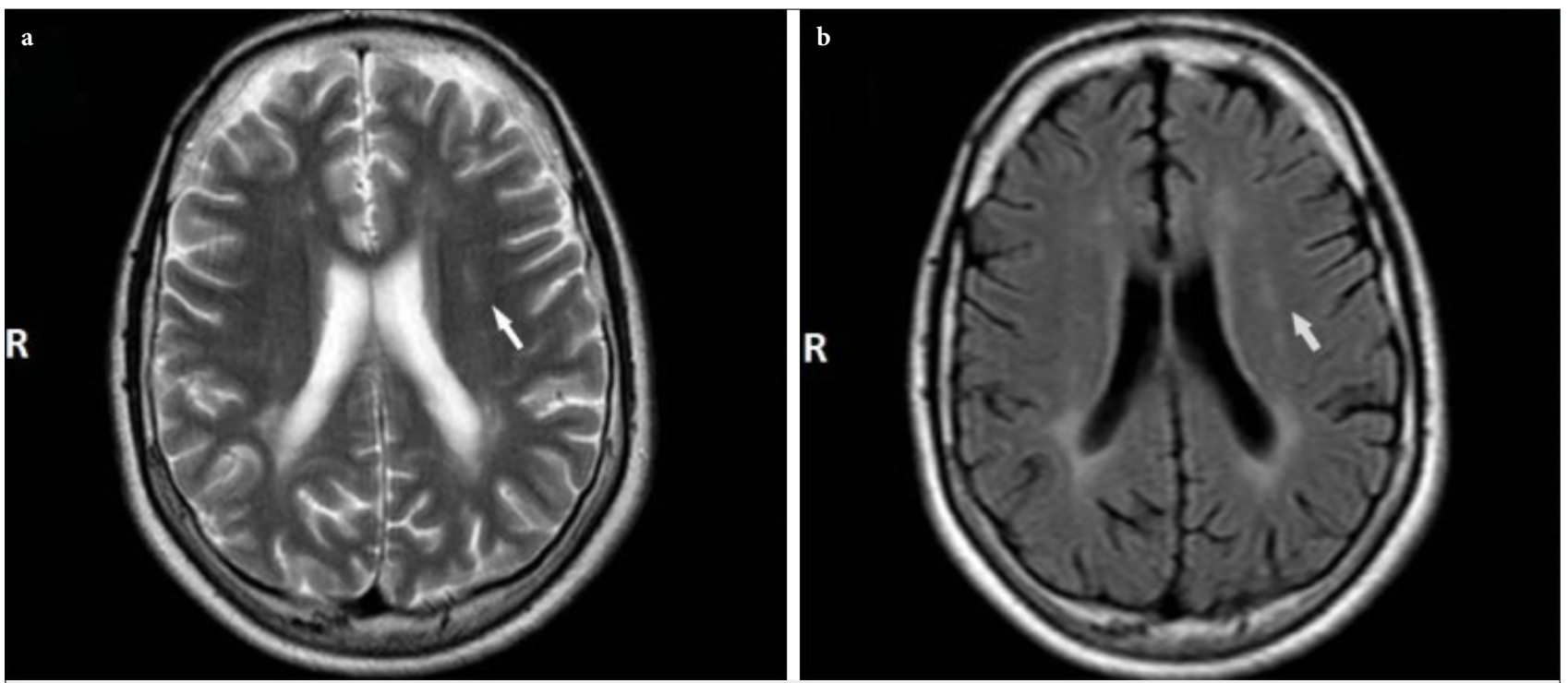

Figure 1. Hyperintense lesions consistent with periventricular leukomalacia surrounding both lateral ventricles in axial MRI scans (a) T2-weighted, (b) FLAIR sequence. 
abnormalities and ventricular enlargement $(11,14,15)$. All of the abovementioned findings suggest that the causality between PVL and schizophrenia cannot be solely attributed to a neurostructural abnormalityschizophrenia nexus, and that the shared multifactorial etiology including neuroinflammation that leads to both pathologies, should be comprehensively examined to provide a deeper appreciation of the underpinnings of the involvement of impaired neurodevelopmental processes in the schizophrenia etiopathogenesis.

Informed Consent: All procedures followed were under the ethical standards stated in the Helsinki Declaration of 1975 (in its most recently amended version). Informed consent was obtained from the patient after explaining to him why we request his permission to use his clinical information in a scientific work.

Conflict of Interest: The authors declared no potential conflicts of interest with respect to the research, authorship, and/or publication of this article.

Financial Disclosure: The authors received no financial support for the research, authorship, and/or publication of this article.

\section{REFERENCES}

1. Owen MJ, Sawa A, Mortensen PB. Schizophrenia. Lancet 2016; 388:86-97.

2. Keshavan MS, Collin G, Guimond S, Kelly S, Prasad KM, Lizano P. Neuroimaging in Schizophrenia. Neuroimaging Clin N Am $2020 ; 30: 73-83$.

3. Balcioglu YH, Berkol TD, Cevik FE, Oncu F, Ozgen G. Electroconvulsive therapy in schizophrenia with coincidental choroidal fissure cyst: a case report. J Neurobehav Sci 2017; 4:134-137.

4. Balcioglu YH, Kirlioglu SS, Berkol TD, Ozgen G. Coincidental mega cisterna magna with psychotic disorder: a possible neuroanatomical liability for a shared psychotic disorder. Anatolian Journal of Psychiatry 2018; 19-:106-110.
5. Gilmore JH, Castillo M, Rojas M. Early onset schizophrenia in a patient with premature birth, germinal matrix hemorrhage and periventricular leukomalacia. Schizophr Res 2000; 44:158-160.

6. Folkerth RD. Periventricular leukomalacia: overview and recent findings. Pediatr Dev Pathol 2006; 9:3-13.

7. Cosgrove D, Mothersill O, Kendall K, Konte B, Harold D, Giegling I, et al. Cognitive Characterization of Schizophrenia Risk Variants Involved in Synaptic Transmission: Evidence of CACNA1C's Role in Working Memory. Neuropsychopharmacology 2017; 42:2612-2622.

8. Leviton A, Gilles F. Ventriculomegaly, delayed myelination, white matter hypoplasia, and "periventricular" leukomalacia: how are they related? Pediatr Neurol 1996; 15:127-136.

9. Dammann O, Leviton A. Infection remote from the brain, neonatal white matter damage, and cerebral palsy in the preterm infant. Semin Pediatr Neurol 1998; 5-:190-201.

10. Marín-Padilla M. Three-dimensional structural organization of layer I of the human cerebral cortex: a Golgi study. J Comp Neurol 1990; 299:89-105.

11. Thomas KL, Huttunen M, Marin-Padilla N. Neural migration, pro-inflammatory interleukins and periventricular leukomalacia: Involvement in schizophrenia [2] (multiple letters). Trends Neurosci 1999; 22:389-390.

12. Huang J, Zhang L, Kang B, Zhu T, Li Y, Zhao F, Qu Y, $\mathrm{Mu}$ D. Association between perinatal hypoxic-ischemia and periventricular leukomalacia in preterm infants: A systematic review and meta-analysis. PLoS One 2017; 12:e0184993.

13. Giannopoulou I, Pagida MA, Briana DD, Panayotacopoulou MT. Perinatal hypoxia as a risk factor for psychopathology later in life: the role of dopamine and neurotrophins. Hormones (Athens) 2018; 17:25-32.

14. Balcioglu YH, Kirlioglu SS. C-Reactive Protein/Albumin and Neutrophil/Albumin Ratios as Novel Inflammatory Markers in Patients with Schizophrenia. Psychiatry Investig 2020; 17:902-910.

15. Walton E, Hibar DP, van Erp TGM, Potkin SG, Roiz-Santiañez R, Crespo-Facorro B, et al. Prefrontal cortical thinning links to negative symptoms in schizophrenia via the ENIGMA consortium. Psychol Med 2018; 48:82-94. 